\title{
LOCALIZACIÓN Y ACCESO AL VERDE URBANO DE LA CIUDAD DE SALAMANCA
}

\author{
Alejandro Gómez Gonçalves \\ Departamento de Geografía. Universidad de Salamanca \\ algomez@usal.es
}

\section{RESUMEN}

El objetivo de este artículo es evaluar el estado del verde urbano de la ciudad de Salamanca. La metodología empleada ha permitido seleccionar los espacios de mayor tamaño, que son los que concentrarían un mayor número de visitas. Utilizando su posición en la ciudad, se ha estimado el número de personas que no tienen acceso a la trama verde. El análisis histórico confirma la utilidad del verde urbano para identificar las deficiencias en la expansión urbana y para caracterizar un modelo de ciudad propio del sur de Europa, donde la mayoría de las áreas verdes han surgido en fechas recientes.

Palabras clave: verde urbano, ciudad, espacios verdes, geografía urbana, Salamanca.

\begin{abstract}
Localization and accessibility to urban green spaces in Salamanca (Spain). The aim of this paper is to evaluate the state of urban green spaces in the city of Salamanca. The chosen methodology has allowed the selection of larger areas because they could concentrate a greater number of visits. The number of people without access to urban parks has been estimated using their position in the city. The historical analysis confirms the value of urban green spaces to identify deficiencies in the urban sprawl and to characterize the typical city patterns of southern Europe, where most green areas have been created recently.
\end{abstract}

Key words: urban green spaces, city, green areas, urban geography, Salamanca.

Fecha de recepción: enero 2012.

Fecha de aceptación: noviembre 2012. 


\section{INTRODUCCIÓN Y OBJETIVOS}

Los espacios verdes constituyen uno de los componentes más representativos del medio ambiente urbano, presentando una dualidad de partida, en la medida en que su funcionamiento responde a leyes biológicas, mientras que su aparición está en muchos casos vinculada al cumplimiento de objetivos sociales (Corona, 2001). El verde urbano está integrado mayoritariamente por parques y jardines públicos, cuya gestión y mantenimiento dependen de las distintas administraciones. A lo largo del siglo XVIII y del XIX surgieron grandes parques en las principales ciudades europeas y americanas, como Central Park en Nueva York, Hyde Park en Londres, Vondelpark en Ámsterdam, el Parque del Retiro en Madrid o el Bois de Boulogne en París, ya que los distintos estados se vieron obligados a asumir responsabilidades en la configuración de la ciudad para mejorar las condiciones de vida de sus habitantes. Hoy en día, la incorporación de las áreas verdes al planeamiento urbano se ha convertido en un derecho de los ciudadanos (Sanesi y Chiarello, 2006) consagrado en la normativa urbanística, a medida que los beneficios que generan van siendo demostrados científicamente.

En efecto, las funciones desempeñadas por el verde urbano se pueden clasificar en cinco grandes grupos: ecológicas, económicas, sociales, de planificación urbana y transversales (Baycan-Levent et al., 2004). Sin entrar a valorar en detalle cada una de ellas, conviene al menos señalar la importancia de los espacios verdes para la habitabilidad de las ciudades, ya que ejercen de purificadores del aire al absorber la polución atmosférica (McPherson et al., 1994; Bolund y Hunhammar, 1999; Priego-González, 2008), secuestrando el $\mathrm{CO}_{2}$ atmosférico (Nowak y Crane, 2002) y reduciendo el ozono troposférico (Dwyer et al., 1992; PriegoGonzález, 2008). Además actúan como reguladores del clima local (Akbari et al., 1992; McPherson et al., 1994; Ochoa, 2009), lo que puede llegar a provocar un sensible ahorro de energía en determinadas ocasiones. La trama verde es una herramienta fundamental para la configuración de la ciudad por su destacada capacidad para organizar el espacio urbano y en ocasiones se puede llegar a convertir en un aliciente para los negocios y para el turismo. Dentro de las funciones sociales, el verde urbano induce notables beneficios terapéuticos en los habitantes, ayudándolos a recuperarse del estrés diario (Ulrich et al., 1991; Galindo et al., 1997; Bolund y Hunhammar, 1999), al tiempo que permiten mejorar la relación social y personal de los ciudadanos (Bendimo-Rung et al., 2005). Habría que añadir otros potenciales beneficios para la salud como son la reducción de la obesidad y de enfermedades coronarias vinculada a las actividades llevadas a cabo en el verde urbano, así como la mitigación del tipo de vida sedentario (De Vries et al., 2003; Santana et al., 2007). Sánchez-Barbudo (1989) considera las áreas verdes como un elemento destacado en la educación, debido a la capacidad que tiene el medio ambiente local para motivar a los niños, sirviendo de puente entre el aula y la naturaleza.

Sin embargo, las funciones desempeñadas por el verde urbano no pueden ser disfrutadas de igual manera por todos los habitantes por la irregular distribución de estos espacios en el interior de la trama urbana. Este artículo se ha propuesto analizar las carencias en materia de áreas verdes de una ciudad española de tamaño intermedio, realizando una estimación del número de personas que tendrán dificultades para hacer uso de las mismas. Para ello ha sido necesario establecer una definición de verde urbano en el punto dedicado a la metodología, precisando claramente qué es una ciudad y determinando el tamaño mínimo que debe 
tener un espacio verde para que pueda ser visitado por los ciudadanos. Otro objetivo de este artículo ha sido realizar una revisión de la evolución histórica de estos equipamientos que permitiera identificar las razones que se encuentran detrás de las actuales deficiencias en el acceso a las zonas verdes registradas en determinados barrios de la ciudad. Este análisis se ha desarrollado en el tercer punto del artículo y ha permitido identificar el periodo y las causas de aparición de los elementos que conforma la trama verde salmantina. En el cuarto punto se ha determinado el acceso de la población al verde urbano, identificando las partes de la ciudad que presentan mayores carencias en este tipo de dotaciones. La investigación se ha llevado a cabo en la ciudad de Salamanca, situada en la submeseta norte de la Península Ibérica, a orillas del río Tormes, uno de los principales afluentes meridionales del Duero. Según los datos del INE, el municipio contaba en 2011 con una población de 153.472 habitantes, siendo capital de una de las nueve provincias que forman la Comunidad Autónoma de Castilla y León.

\section{METODOLOGÍA}

\section{1. ¿Cómo se seleccionó el verde urbano de Salamanca?}

Verde urbano, parques, jardines, áreas verdes y sus numerosos sinónimos son términos socialmente muy utilizados para los que no existe una definición común desde un punto de vista científico. Por tanto, una investigación que acometa el estudio de estos espacios en el interior de la ciudad debe proponer una definición clara y justificada. En este artículo se ha empleado el término de verde urbano y de forma previa a su definición se ha delimitado la ciudad o lo urbano, seleccionando posteriormente una serie de zonas con vegetación localizadas en su interior que pudieran ser visitadas por los ciudadanos.

Intentar establecer los límites de una ciudad es una tarea compleja porque no existe consenso para fijar las fronteras del fenómeno urbano. Al tratarse en este caso de una entidad urbana de tamaño intermedio, se optó por una técnica con la que delimitar el núcleo central ya que, pese a las nuevas formas urbanas, en el interior de las aglomeraciones todavía puede distinguirse el lugar central donde se concentran la mayor parte de las residencias y de los puestos de trabajo (Roca, 2003). Por tanto, se ha optado por reducir la ciudad al municipio vertebrador del área urbana, utilizando la división administrativa actual y se ha empleado también un criterio morfológico citado por Nel·lo y Muñoz (2004), con el que se integran en una misma aglomeración de espacio construido todas las parcelas que presenten discontinuidades espaciales inferiores a $200 \mathrm{~m}$ de suelo no urbano.

Mediante un programa de SIG, en concreto el ArcView 3.2, se amplió la imagen final de la ciudad hasta hacer coincidir sus límites con los bordes verticales y horizontales de un A-4. La utilización de un marco fijo, como es un A-4, para la representación gráfica de una urbe implica que cuanto más grande es el polígono urbano, más pequeña será entonces la escala y por lo tanto, mayor será el divisor. En esta investigación se ha empleado el tamaño de la ciudad para determinar la dimensión mínima del verde urbano y para ello se ha utilizado un coeficiente de multiplicación fijo, para poder así comparar estudios realizados en ciudades diferentes. Como se trata de la primera vez que se emplea un método de este tipo, se optó por testar varios factores de multiplicación en diferentes ciudades de Castilla y León próximas a 
Salamanca y al analizar los resultados obtenidos se escogió un coeficiente de $0,3 \mathrm{~m}^{2}$, que se consideró como el más adecuado. Las principales ventajas de esta técnica son la superación de los inconvenientes generados por la utilización de un tamaño mínimo común del verde urbano en ciudades de distinto tamaño, al poner en relación la dimensión de los espacios verdes con la de la urbe en el que están ubicados, y la inclusión de todas las zonas verdes de la ciudad independientemente de la entidad encargada de su mantenimiento. En el caso de la ciudad de Salamanca la escala obtenida mediante este proceso fue de 1/30.000, que al multiplicarse por el factor de $0,3 \mathrm{~m}^{2}$ estableció la superficie mínima del verde urbano en 0,9 ha.

Las ortofotos de 2007 se obtuvieron de la web del Instituto Técnico Agrario de Castilla y León (htttp://www.itacyl.es) y se utilizaron para delimitar la ciudad y el verde urbano. Dentro de esta última categoría se incluyeron aquellas zonas con una superficie igual o mayor a la obtenida mediante la operación anteriormente referida. Para analizar la posibilidad de uso de estos espacios se ha utilizado el concepto de verde urbano disponible, que se define como el conjunto de áreas verdes que pueden ser visitadas por la mayor parte de los ciudadanos al no presentar un carácter privado ni una configuración inaccesible. En la categoría genérica de verde urbano se incluyeron de forma complementaria huertos urbanos y las zonas verdes no disponibles, puesto que desarrollan funciones beneficiosas para los seres humanos, al contribuir a la reducción del estrés y a la mejora de la calidad de vida (Bolund y Hunhammar, 1999).

\section{2. ¿Qué espacios forman parte del verde urbano?}

Como el objetivo de esta investigación es analizar la posibilidad de uso de las áreas verdes por parte de la población, se ha prestado especial atención a los espacios con un tamaño suficiente para desempeñar alguna de las funciones tradicionales como son el ocio, el deporte, el paseo, etc. (Canosa et al., 2003). Las grandes áreas verdes serían las que teóricamente recibirían más visitas debido a que el tamaño se encuentra en relación con la cantidad de equipamientos que suelen poseer, contribuyendo así a satisfacer una mayor variedad de necesidades humanas (Van Herzele y Wiedemann, 2003).

No se incluyeron las zonas verdes de menores dimensiones por no ser capaces de atraer tantos potenciales usuarios como las de gran tamaño, prescindiendo de los parterres de césped, jardineras e incluso de vegetación espontánea en tejados y solares aislados, así como de aquellas no frecuentadas debido a su especial localización, como ocurre con las rotondas.

Para obtener el tamaño mínimo que un espacio debería tener para ser incluido en la categoría de verde urbano se testaron diversas técnicas hasta obtener la más adecuada para desarrollar una investigación de estas características. En primer lugar se recurrió a lo que en principio parece más lógico si se pretende trabajar con las áreas verdes de una ciudad, es decir, los datos de las zonas verdes que gestionan los gobiernos locales. Utilizando el censo proporcionado por el Servicio de Parques y Jardines del Ayuntamiento de Salamanca (SPYJAS), se ordenaron los espacios gestionados por este organismo, en un ranking atendiendo a su superficie (ver Figura 1), lo que permitió comprobar que con un número reducido de los parques de mayor tamaño se obtienen cifras porcentualmente muy significativas del total de áreas verdes censadas. Sin embargo, estos datos presentan un grave inconveniente para realizar un estudio como el aquí propuesto, al incluir espacios situados fuera del perímetro de lo que en esta investigación se ha considerado como ciudad. Además, algunas zonas 
verdes que forman un continuo espacial han sido divididas en varios sectores por motivos ligados a la optimización de su gestión y áreas de dimensiones reducidas han sido agrupadas creando una categoría de verde denominada «barrio». No aparecen los espacios verdes que no son gestionados por el ayuntamiento, aunque las instituciones encargadas de su manejo sean públicas, pero en cambio sí se incluyen los no visitados como son las glorietas. Por todo ello se consideró que los datos proporcionados por los ayuntamientos no eran suficientes para cumplir con los objetivos planteados.

Figura 1

PORCENTAJE ACUMULADO DE LA SUPERFICIE VERDE TOTAL GESTIONADA POR EL AYUNTAMIENTO DE SALAMANCA

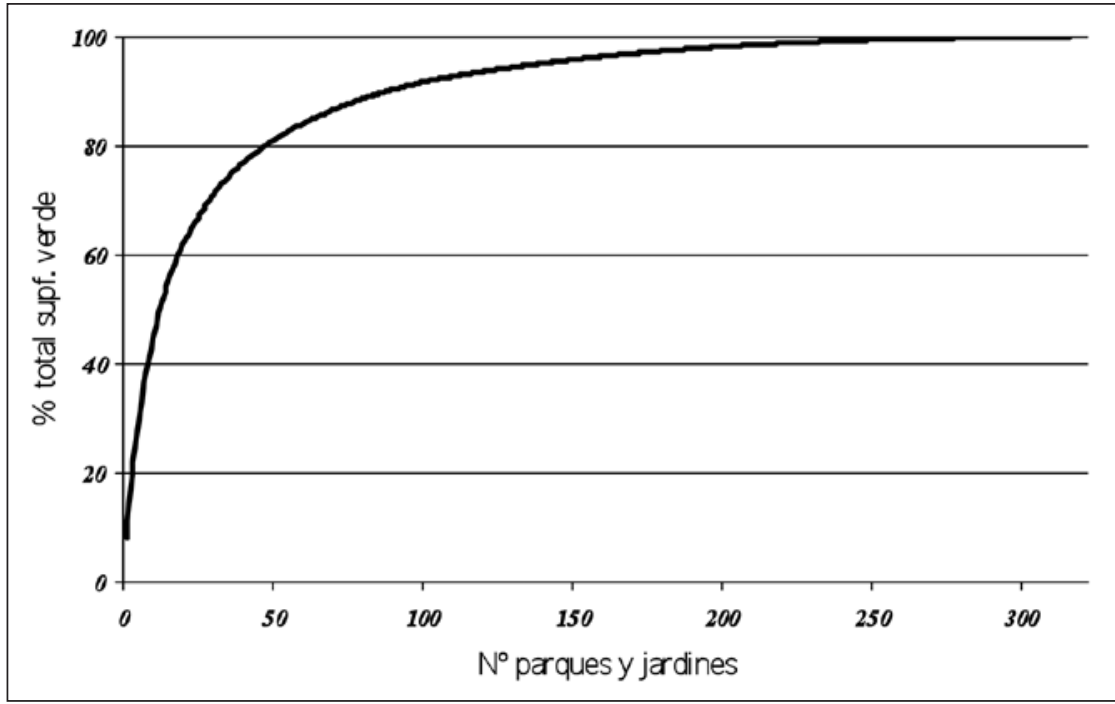

Fuente: elaboración propia a partir de los datos del SPYJAS.

Posteriormente se consultaron varias publicaciones con una temática similar y que afrontaron el reto de establecer una superficie mínima fija para incluir un espacio con vegetación dentro de las diferentes categorías utilizadas para referirse al verde urbano. En España, Canosa et al. (2003) trabajaron con municipios de distinto tamaño demográfico, lo que les obligó a buscar una superficie mínima de las áreas verdes válida para trabajar en urbes de distinta dimensión. Utilizaron un valor fijo de 4 hectáreas como límite para incluir una zona verde dentro de la categoría de parque urbano, independientemente del tamaño de cada municipio. Este criterio fue válido para el estudio llevado a cabo en la Comunidad de Madrid, pero no resultó operativo al ser aplicado en la ciudad de Salamanca, ya que provocaría alteraciones significativas en los resultados al excluir una parte considerable de la trama verde. Con el objetivo de establecer una dimensión mínima para el verde urbano, se revisó la normativa urbanística actualmente en vigor en la ciudad objeto de estudio, esto es, la Ley de Urbanismo de Castilla y León de 1999 y su Reglamento (RUCYL) modificado en 2009. Este último presenta una definición de zonas verdes un tanto ambigua, pero posee un valor 
operacional indiscutible para la reserva de espacios libres públicos en los núcleos urbanos, determinando el tamaño mínimo legal de éstos a través del cumplimiento de dos criterios: por un lado obliga a que en el planeamiento general se incluya el sistema general de espacios libres públicos con una superficie mínima de $5 \mathrm{~m}^{2}$ por habitante (artículo 83), y por otro lado exige una reserva de suelo para el sistema local de espacios libres públicos que debe alcanzar entre el $15 \%$ y el $20 \%$ de los metros cuadrados construibles (artículo 105). Por lo tanto, parecería razonable poner en relación el tamaño mínimo de las zonas verdes con la dimensión de la ciudad en la que se encuentren, puesto que el número de habitantes y el tamaño de las nuevas piezas urbanas determinan la superficie mínima de la trama verde. El resultado obtenido permitiría comparar entre sí ciudades con dimensiones territoriales y demográficas muy diferentes, y reduciría el elevado número de parques y jardines que gestionan las administraciones locales para poder trabajar únicamente con los espacios verdes de mayor tamaño.

Para valorar la posibilidad de uso del verde urbano por parte de los ciudadanos, se ha empleado la distancia máxima que una persona estaría dispuesta a recorrer a pie desde su lugar de residencia hasta llegar a ellos. Varias publicaciones han corroborado que la distancia desde el domicilio habitual es el determinante principal de las visitas a las áreas verdes (Van Herzele y Wiedemann, 2003; Santana et al., 2010), por lo que su utilización permitirá determinar qué parte de la ciudad posee acceso a dichos espacios. En la Conferencia de las Naciones Unidas sobre Medio Ambiente y Desarrollo Sostenible celebrada en Río de Janeiro en 1992 se crearon las Agendas 21 locales, con las que se pretendía incorporar el desarrollo sostenible a las políticas municipales. A nivel europeo, los Acuerdos de Río fueron seguidos por la Carta de Aalborg en 1994 y en 1996 por el informe «Ciudades europeas sostenibles». En 2003 la Comisión Europea publicó el informe «Hacia un perfil de la sostenibilidad local. Indicadores comunes europeos» con el fin de suministrar información objetiva y comparable de la sostenibilidad en las ciudades. En este documento se elabora la Primera Generación de Indicadores Comunes Europeos y entre los cinco indicadores principales aparece la existencia de zonas verdes públicas y de servicios locales, cuya evaluación se realiza a través del cálculo del área de influencia de dichas dotaciones. Nilsson et al. (1997) citan estudios que demostraron que una de cada cuatro personas pospone una visita diaria al verde urbano cuando la distancia desde el lugar de residencia supera los 300 metros, apuntando que cuanto más grande sea la distancia, mayor será el número de personas que posponga alguna visita. En la Primera Generación de Indicadores Comunes Europeos se utilizan 300 metros como distancia mínima para delimitar el área de influencia del verde urbano, con la particularidad de que sólo se tienen en cuenta los espacios con más de 0,5 ha. Sin embargo, no existe un acuerdo en la literatura consultada ya que otras publicaciones emplean una distancia de 400 metros desde las zonas verdes (Magalhães, 1992; Van Herzele y Wiedemann, 2003; Santana et al., 2007). En este artículo se ha optado por utilizar esta última cifra por considerarla como integradora, tratando con ello de asegurar que en las zonas sin acceso los ciudadanos posponen un elevado número de visitas a los espacios verdes.

Como se mencionó anteriormente, se ha fijado la superficie mínima del verde urbano en función del tamaño de la ciudad y en el caso de Salamanca ha dado como resultado 0,9 ha, aunque para determinar el acceso solamente se han utilizado los espacios que pueden ser visitados por los ciudadanos. No obstante, la trama verde total de la ciudad en 2007 estaba compuesta por treinta y cuatro zonas verdes de las que ocho eran verdes urbanos no disponibles, y se optó por añadir 
los Jardines del Hospital Clínico que poseían 0,82 ha (ver Figura 2). En el estudio histórico de su construcción, en buena lógica, se han contemplado, en cada periodo, todos aquellos jardines y huertos privados que con el tiempo se convirtieron en espacios verdes de uso público.

\section{3. ¿Cómo se estudió el acceso al verde urbano disponible de Salamanca?}

El tamaño de un área verde puede modificar la distancia que una persona está dispuesta a recorrer, puesto que los grandes parques son capaces de satisfacer un mayor número de necesidades humanas (Van Herzele y Wiedemann, 2003). Aceptando las propuestas de estos autores, se ha empleado una distancia media de 800 metros desde las áreas verdes con una extensión superior a 10 ha, mientras que en las que poseen un tamaño inferior se mantuvieron los 400 metros.

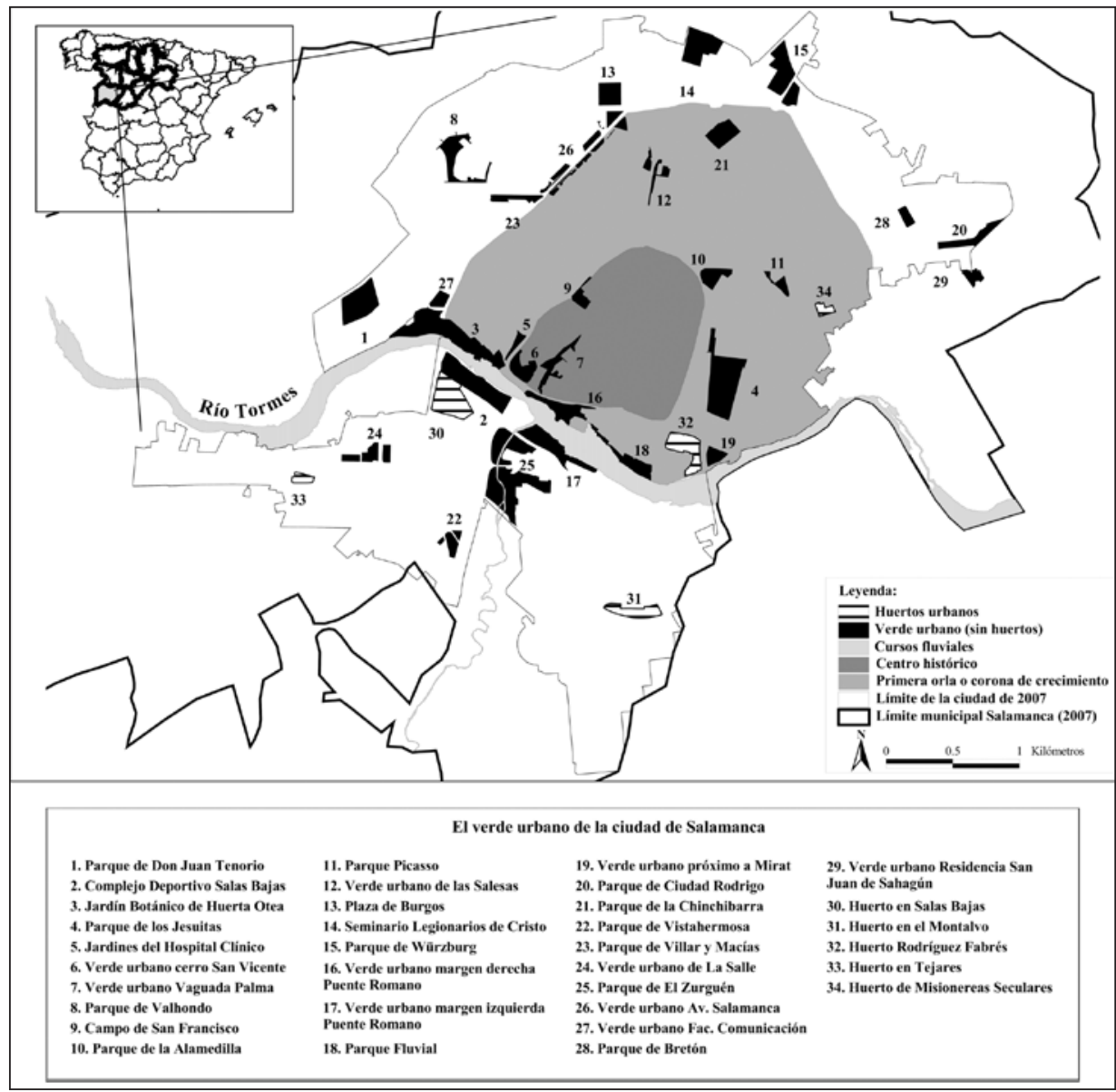

Fuente: elaboración propia a partir de la ortofotografía de 2007 del Itacyl. 
Estos indicadores se han completado añadiendo la distancia máxima de 100 metros desde los espacios verdes con equipamientos infantiles, con la que teóricamente se cubrirían las necesidades de familias con niños de entre 0 y 5 años (Santana et al., 2010). La construcción de este tipo de dotaciones aparece recogida en el artículo 105 del RUCYL, al menos en los espacios libres públicos creados por el planeamiento local, y obliga a reservar áreas especiales dedicadas al juego infantil con unas características mínimas.

Para determinar las partes de la ciudad con acceso al verde urbano disponible se empleó el buffering, una operación de proximidad de los SIGs vectoriales que consiste en generar polígonos por una condición de proximidad a un conjunto de elementos geográficos, en este caso las áreas verdes urbanas, obteniéndose así el espacio dentro del cual los ciudadanos tendrían acceso a estas dotaciones. La población que no posee acceso a las zonas verdes se ha estimado a través de un análisis por barrios, determinando el porcentaje de la superficie sin acceso en cada uno de los cuarenta y cinco barrios con los que contaba Salamanca en 2008 y multiplicándolo por los residentes en estas unidades urbanas.

\section{4. ¿Cómo se analizó la evolución del verde urbano en la ciudad de Salamanca?}

El estudio de la evolución de la ciudad y de las áreas verdes en su interior, se ha realizado mediante la identificación del periodo de aparición de los espacios que conformaban la trama verde de Salamanca en el año 2007. De entre la variedad de documentos cartográficos existentes, los que recogen más fielmente la realidad urbana en cada momento son las fotografías aéreas y las ortofotografías, debido a que ofrecen una imagen puntual del tejido urbano y permiten obtener de una manera exacta la delimitación de la ciudad. Se han utilizado tres fotografías aéreas, la de 1956, 1971 y 1984, y dos ortofotografías, de 2002 y de 2007. A través de estos documentos se desarrolló una valoración real, y con gran exactitud, de la expansión territorial de la ciudad y de la configuración de la trama verde a lo largo de los últimos cincuenta años. Se generaron distintos documentos cartográficos a partir del trazado de los límites de la ciudad en cada uno de los años mencionados, reconstruyendo la evolución histórica de Salamanca y de sus principales acontecimientos urbanísticos, para lo que se utilizó como apoyo la literatura especializada en esta cuestión. En el análisis de la evolución urbana se utilizó el barrio como la unidad administrativa de referencia debido a su tamaño intermedio, a que no son tan extensos como los distritos ni tan reducidos como las manzanas o las circunscripciones electorales, a su carácter más o menos estable en el tiempo y a su fácil identificación por parte de los ciudadanos.

Se han diferenciado cuatro sectores de la ciudad: el centro histórico, compuesto por los barrios situados en el interior de la cerca medieval, la primera orla o corona de crecimiento, que comprende una franja de terreno adyacente al centro histórico cuyo límite meridional lo establece el río, la segunda orla o corona de crecimiento, que estaría compuesta por todo el territorio exterior a las dos unidades anteriormente citadas, y en cuarto lugar, los espacios situados al sur del Tormes. La fecha final de este trabajo se fijó en 2007, año de la publicación del nuevo Plan General de Ordenación Urbana, y supone un análisis de conjunto del urbanismo en Salamanca previo al hundimiento del sector inmobiliario. 


\section{EVOLUCIÓN HISTÓRICA DEL VERDE URBANO SALMANTINO}

\subsection{Aparición de áreas verdes hasta 1956}

El surgimiento de los primeros espacios verdes públicos en la ciudad está vinculado al cumplimiento de una serie de medidas ambientalistas que perseguía una mejora en la ventilación y en la higiene de Salamanca. Desde finales del siglo XVIII se produjo el derribo progresivo de la muralla y de sus puertas, siendo sustituidas por paseos arbolados. Sin embargo, habrá que esperar hasta las primeras décadas del siglo XIX para encontrar el origen del verde urbano actual, ya que el Campo de San Francisco, situado en el límite occidental de la cerca medieval, se convirtió en parque en 1828 y la Alamedilla, adyacente al extremo nororiental, lo hizo en 1884. A comienzos del siglo XX Salamanca era una ciudad que contaba con poco más de veinticinco mil habitantes y que en su condición de centro económico provincial, ejercía cierto atractivo sobre la población rural en busca de una salida a las penurias de la vida en el campo. Durante los años treinta la falta de recursos dificultó el acceso de una gran parte de la población al mercado de vivienda, motivando la actuación de la iniciativa pública. Desde mediados de la década de los cuarenta comenzó la construcción de vivienda social en Salamanca a través del Instituto Nacional de la Vivienda, organismo creado por el Estado en 1939 y que dependía del Ministerio de Trabajo. Aparecieron barrios enteros tanto en el sector occidental de la primera orla de crecimiento, como en la margen izquierda del río, siendo complementados por las actuaciones llevadas a cabo por RENFE y por la Caja de Ahorros (Villar y Burrieza, 1995). En 1956 la ciudad había alcanzado una extensión de 618 ha, frente a las 162 que se estima que pudiera tener a principios de siglo, debido al considerable aumento del número de habitantes entre 1920 y 1950.

De las ocho zonas verdes con las que contaba Salamanca (ver Figura 3), seis estaban dedicadas a la agricultura. De entre estas últimas, las pertenecientes a los colegios de las Esclavas, de las Salesas y de los Jesuitas se convertirán en parques públicos durante la década de los setenta y principios de los ochenta. El huerto urbano más grande estaba situado en la margen derecha del Tormes y contaba con más de veintiuna hectáreas de extensión, de las que la mayor parte pertenecían a la Fundación Rodríguez Fabrés.

\subsection{Consecuencias del Plan Maroto y del PGOU de 1966}

A finales de la década de los treinta, se instaló en Salamanca el cuartel general de las fuerzas sublevadas y antes de finalizar la Guerra Civil se redactó el Plan D’Ors-Gamazo. Aunque no llegó a aplicarse, tiene interés debido a que junto a los presupuestos ideológicos del Movimiento, heredaba ciertos principios del urbanismo ambientalista que se plasmaron en propuestas para la creación de un cinturón verde en torno a la ciudad y de un gran parque en el centro histórico. En 1944 se aprobó el llamado Plan Maroto y en 1966 fue sustituido por un Plan General que trataba de limitar la expansión de la ciudad y de concretar un moderno sistema de comunicaciones (Senabre, 2003a). Estas pretensiones se vieron desbordadas por un intenso crecimiento, tanto demográfico como territorial, originado por la llegada de un importante contingente de población rural procedente en su mayor parte de la provincia de Salamanca. Aparecieron nuevos barrios y desde los años setenta comenzó a configurarse un área urbana en torno a la capital, 
compuesta por pequeños núcleos de población. Durante este último periodo el consistorio realizó una aproximación simplemente formal a la normativa urbanística recogida en la Ley del Suelo de 1956, redactando una serie de Planes Parciales que no llegaron a aprobarse y que además no cumplían con las exigencias mínimas de dotaciones urbanísticas (Senabre, 2003a).

Desde comienzos de la década de los setenta y con la formación de los primeros ayuntamientos democráticos, se asistirá a una serie de actuaciones dirigidas a mitigar las deficiencias dotacionales en determinados barrios de la ciudad, entre las que se incluye la adquisición de jardines y huertos situados en el interior de la trama urbana y que pertenecían a diversas órdenes religiosas. Hasta 1984 se producirá la conversión de tres de estos huertos urbanos en parques públicos y la construcción de una serie de edificios religiosos en la periferia de la ciudad que darán como resultado la aparición, a medio plazo, de otros tres espacios verdes. También se crearon el Parque Fluvial en la margen derecha del Tormes, en una zona históricamente dedicada a labores agrícolas, el pequeño Parque de Garrido, en el norte de la ciudad, y los Jardines del Hospital Clínico, que fue inaugurado en 1975. Además, el crecimiento espacial de la ciudad provocó que quedasen enclavados en el tejido urbano dos huertos en terrenos adyacentes a la vía férrea en el sur de la ciudad.

\subsection{Expansión de la trama verde con el PGOU de 1984}

Figura 3

\section{EVOLUCIÓN DEL VERDE URBANO DE SALAMANCA}

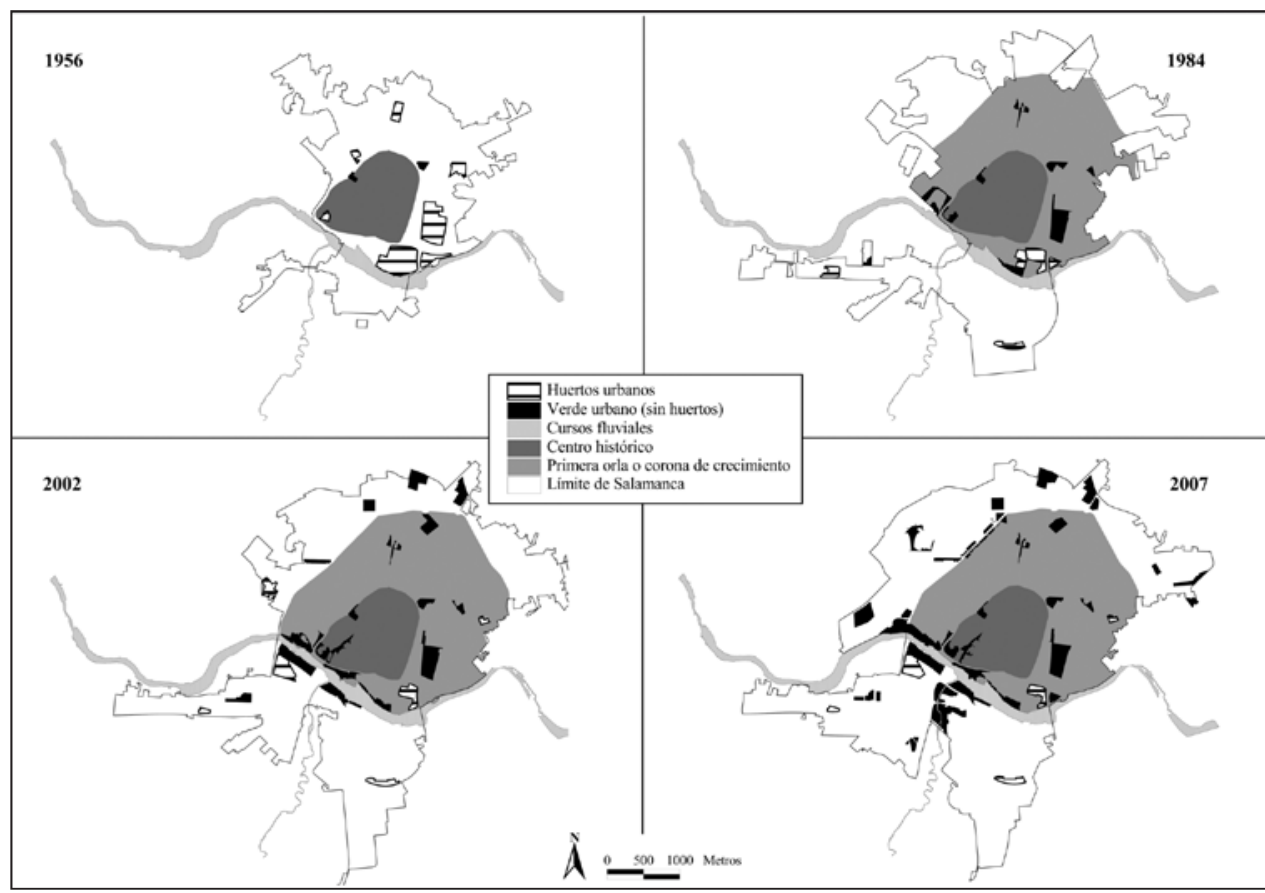

Fuente: elaboración propia a partir de las fotografías aéreas de 1956 del CECAT y de 1984 del CNIG, de las ortofotografías de 2002 y de 2007 del Itacyl, y del Ayuntamiento de Salamanca. 
En 1984 se aprobaron dos documentos claves en el planeamiento urbano de Salamanca: el Plan General de Ordenación Urbana y el Plan Especial de Protección y Reforma Interior del Recinto Universitario y Zona Histórico-Artística (PECH). Ambos propiciaron una ocupación de los espacios urbanos hasta entonces vacíos y fomentaron la protección y la revitalización del centro histórico. La UNESCO declaró el casco antiguo de Salamanca como Patrimonio de la Humanidad en 1988, impulsando decididamente las intenciones del PECH en materia de protección y recuperación de la zona monumental. El nuevo Plan General incorporó las exigencias de la Ley del Suelo de 1976, provocando un cambio notable en la forma de planificar la ciudad que propició una reserva sistemática de suelo para espacios libres públicos en las nuevas piezas urbanas. Durante los veintitrés años en los que estuvo en vigencia el Plan General se crearon veintidós verdes urbanos a lo largo de dos etapas de distinta duración, para cuya separación se toma como referencia la ortofoto de 2002, año en que se celebra en Salamanca la capitalidad europea de la cultura.

\subsubsection{De $1984-2002$}

Durante este periodo se produjo estancamiento demográfico del municipio de Salamanca, mientras que los núcleos periféricos emprendieron un crecimiento que se mantiene a día de hoy. Como contraste, la ciudad experimentó una progresiva expansión territorial, especialmente intensa en el sur del río Tormes, donde se llevaron a cabo intervenciones de considerable envergadura como fueron la ampliación del polígono industrial de El Montalvo en 1991, la aprobación del Plan Parcial Alambres-Vistahermosa un año después y el comienzo de la construcción del barrio del Zurguén en 1994 (Hortelano, 2000). Con la apertura del Palacio de Congresos en 1992 y la creación de un verde urbano a su alrededor, se asistió a una progresiva reconversión del cuadrante suroccidental del centro histórico, que indujo una paulatina mudanza de los pobladores dejando paso a personas con mayores rentas. En esta primera etapa del periodo de vigencia del PGOU de 1984, fueron creados catorce verdes urbanos al incorporar al nuevo planeamiento las exigencias urbanísticas en materias dotacionales, de tal manera que en cada uno de los nuevos barrios se reservó suelo para espacios verdes. Al mismo tiempo se intentaron mitigar las carencias existentes, aunque se trata siempre de una labor compleja por la dificultad de intervenir en la trama urbana consolidada. Con esta intención se proyectó el Parque de Wurzbürg en el norte de la ciudad, con el que el Plan General trataba de paliar la falta de espacios verdes de suficiente entidad en uno de los barrios más densamente poblados. Sin embargo, la actuación más destacada fue la realizada en el entorno del río mediante el Plan Especial de Recuperación de las Márgenes del Tormes, donde se combinó la recuperación urbanística y la dotación de zonas verdes. En la margen derecha se demolieron numerosos edificios entre los que se encontraban las antiguas tenerías y se crearon amplias áreas verdes manteniendo pequeñas formaciones vegetales de ribera. En la izquierda se aprovecharon vacíos existentes para diseñar un verde urbano en torno al Puente Romano, junto al que la Universidad de Salamanca construyó un complejo deportivo de grandes dimensiones. Debido al fuerte crecimiento en superficie de la ciudad se incorporaron a la trama verde tres huertos y los jardines del Seminario de los Legionarios de Cristo, situado en el límite septentrional. 
Dentro del municipio de Salamanca y fuera de lo que en este trabajo ha sido considerado como ciudad se produjo, a finales de 2001, la agregación de la finca de la Aldehuela de los Guzmanes, hasta entonces perteneciente a Cabrerizos. Es necesario destacar este hecho porque en ella se ubica el parque más grande del municipio, que aunque esté ubicado fuera de lo que en este trabajo ha sido considerado como ciudad, es posible que en el futuro pudiese llegar a formar parte del continuo urbano.

\subsubsection{De 2002 a 2007}

El segundo periodo en que se ha dividido la aplicación del PGOU de 1984 se caracterizó por un aumento en la intensidad del crecimiento territorial de la trama urbana salmantina, debido al elevado ritmo en la construcción de vivienda nueva que precedió al comienzo de la crisis inmobiliaria en 2008. Hasta esa fecha se construyeron barrios enteros como el de La Platina, en el sector noroccidental, y se terminó la segunda fase de El Zurguén, en el sur de la ciudad. La mayoría de las nueve áreas verdes aparecidas en este periodo surgieron a través del planeamiento parcial y se localizaron en los nuevos sectores incorporados en la periferia de la ciudad. En torno al eje fluvial del río Tormes se construyó mediante el planeamiento general, una serie de verdes urbanos de grandes dimensiones que terminan por consolidar este sector como un auténtico corredor verde. En la margen izquierda se consolidó la actuación llevada a cabo junto al Puente Romano con el diseño del verde urbano más grande de la ciudad, el del Zurguén, que aprovecha las antiguas huertas en torno al arroyo del mismo nombre extendiéndose hasta el límite sur que supone la vía férrea.

\subsection{Recapitulación}

El Plan General de 1984 supuso un hito en la creación de espacios verdes en Salamanca porque consolidó el planeamiento urbano como instrumento a través del cual dirigir un crecimiento equilibrado. Para poder evaluar la situación actual de la trama verde es necesario recurrir a los índices tradicionalmente empleados en estos casos, como son aquellos que ponen en relación la superficie total del verde urbano con el número de habitantes o con la extensión de una ciudad. En la normativa urbanística no existen unos estándares mínimos claros para el conjunto de la ciudad. El RUCYL obliga, por un lado, a que en el planeamiento general se incluya el sistema general de espacios libres públicos con una superficie mínima de $5 \mathrm{~m}^{2}$ por habitante, mientras que en el caso del planeamiento de desarrollo exige una reserva de suelo para espacios libres públicos que equivale a un porcentaje de la superficie construible de los nuevos sectores urbanos. Podrían tomarse como referencia los patrones utilizados en un país vecino como Portugal, donde la DGOT (Dirección General de Ordenación del Territorio) fija en $10 \mathrm{~m}^{2} /$ hab la estructura verde principal de una ciudad, que a su vez debería estar acompañada por otras zonas verdes situadas fuera de la trama urbana como bosques, parques periurbanos y otros espacios, hasta alcanzar los $40 \mathrm{~m}^{2} / \mathrm{hab}$ en total. En Salamanca, el ratio de verde urbano disponible por habitante se sitúa en $6,41 \mathrm{~m}^{2} / \mathrm{hab}$, una cifra claramente inferior al mínimo establecido en Portugal para el interior de las ciudades. Para alcanzar un índice aceptable sería necesario que contase con un total aproximado de 150 ha de áreas verdes, lo que significa que el verde urbano disponible de 2007 representa dos tercios de la superficie mínima estimada. Si computasen como espacios verdes todos los 
parques y jardines gestionados por el SPYJAS pero no incluidos en esta investigación por estar situados fuera de lo que se ha considerado como ciudad, entre los que se encuentra el Parque de la Aldehuela, sumarían 54 ha más de áreas verdes alcanzando así el valor mínimo por habitante. No obstante, parece más correcto incluir estos espacios dentro de la estructura verde situada en la periferia de la ciudad, para la que existen otros patrones diferentes, ya que no están insertadas en el tejido urbano aunque en ocasiones den servicio a varios municipios.

Tabla 1

ÍNDICES DEL VERDE URBANO SALMANTINO

\begin{tabular}{|c|c|c|c|c|c|}
\hline & 1956 & 1971 & 1984 & 2002 & 2007 \\
\hline$\overline{N^{\circ} \text { espacios verdes }}$ & 8 & 8 & 12 & 24 & 34 \\
\hline $\mathrm{N}^{\circ}$ huertos urbanos & 6 & 5 & 3 & 6 & 5 \\
\hline $\mathrm{N}^{\circ}$ espacios verdes sin huertos & 2 & 3 & 9 & 18 & 29 \\
\hline $\mathrm{N}^{\circ}$ espacios verdes disponibles & 2 & 2 & 8 & 16 & 26 \\
\hline $\mathrm{N}^{\circ}$ habitantes & 80.239 & 125.220 & 167.131 & 156.006 & 155.921 \\
\hline Extensión de la ciudad (ha) & 618 & 746 & 1039 & 1301,6 & 1463 \\
\hline Extensión del verde (ha) & 47,9 & 35,4 & 40,8 & 80,6 & 124,0 \\
\hline Extensión del verde sin huertos (ha) & 2,4 & 3,9 & 25,5 & 61,1 & 109,0 \\
\hline Extensión del verde urbano disponible (ha) & 2,4 & 2,7 & 23,7 & 53,5 & 100,0 \\
\hline Densidad de verde $\left(\mathrm{m}^{2} / \mathrm{hab}\right)$ & 5,97 & 2,83 & 2,44 & 5,16 & 7,95 \\
\hline Densidad verde sin huertos ( $\left.\mathrm{m}^{2} / \mathrm{hab}\right)$ & 0,30 & 0,31 & 1,52 & 3,92 & 6,99 \\
\hline Densidad del verde urbano disponible ( $\left.\mathrm{m}^{2} / \mathrm{hab}\right)$ & 0,30 & 0,22 & 1,42 & 3,43 & 6,41 \\
\hline Densidad verde por superfi cie ( $\left.\mathrm{m}^{2} / \mathrm{ha}\right)$ & 774,6 & 475,1 & 392,8 & 618,9 & 847,6 \\
\hline Densidad del verde urbano disponible por superfi cie $\left(\mathrm{m}^{2} / \mathrm{ha}\right)$ & 39,2 & 51,7 & 245,1 & 469,5 & 745,0 \\
\hline
\end{tabular}

Fuente: elaboración propia a partir de datos del INE. Los datos de población de 1956, de 1971 y de 1984 corresponden a los años 1950,1970 y 1981 respectivamente.

Figura 4

\section{EVOLUCIÓN DE LOS PRINCIPALES ÍNDICES DEL VERDE URBANO SALMANTINO}

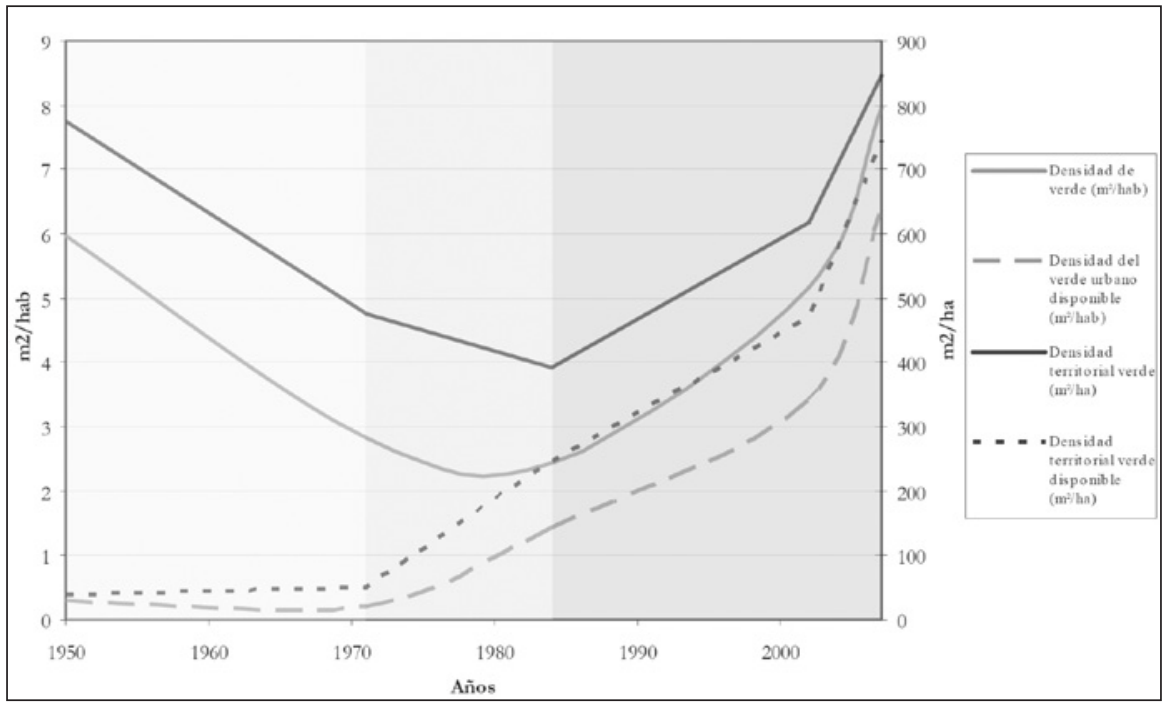

Fuente: elaboración propia a partir de datos del INE. 
Este tipo de índices pueden ocultar situaciones de déficit de espacios verdes en determinadas zonas de la ciudad, al tratarse de valores totales para el conjunto de la misma. Si se quiere evaluar la posibilidad de uso del verde urbano por parte de los ciudadanos, sería necesario complementar esta información con otro tipo de indicadores. No obstante, pueden ofrecer una información muy valiosa del pasado reciente, especialmente de los periodos de mayor crecimiento de las ciudades. Los ratios referentes a zonas verdes por habitante y por hectárea, aparecen en la Tabla 1 y la representación gráfica de su evolución en la Figura 4. Existe una gran diferencia entre las situaciones de partida de los índices referidos al verde urbano disponible y los que recogen la trama verde en su conjunto. Estos últimos presentaban a comienzos del periodo unos ratios similares a los de 2002, debido a que en la década de los cincuenta la agricultura urbana tenía un peso muy importante en el cómputo global, contando con 45,5 ha dedicadas a tal fin. Por otra parte, el índice del verde disponible mantendrá valores muy reducidos hasta alcanzar el mínimo absoluto en 1971. Al no ejecutarse una planificación urbana que reservase suelo para unas mínimas dotaciones durante las décadas anteriores, se siguieron construyendo barrios enteros de vivienda social sin zonas verdes. En dicha fecha, Salamanca contaba con tan solo dos verdes urbanos disponibles, el Campo de San Francisco y el Parque de la Alamedilla, exactamente los mismos que daban servicio a los aproximadamente veinticinco mil habitantes con los que contaba la ciudad a comienzos del siglo XX. Al mismo tiempo, los ratios referentes a la trama verde total sufrieron un descenso debido al fuerte crecimiento urbano y a la reducción de la superficie cultivada en su interior, que quedó limitada en 1984 a una extensión cercana a las 15 ha.

Las actuaciones llevadas a cabo hasta esa fecha supusieron una mejora de los índices del verde disponible al aparecer tres nuevos parques, aunque obviamente, esto no quedase reflejado en los ratios de conjunto. Desde la publicación del PGOU de 1984 se asiste a una tendencia al alza de los valores relativos al verde urbano por habitante y por hectárea, debido al cumplimiento de la normativa urbanística, fundamentalmente en lo referente a la reserva de suelo en las nuevas piezas urbanas. De esta manera al final del periodo de estudio el verde urbano disponible suponía el 88\% de la trama verde total, frente al débil 5\% con el que se inició el periodo de estudio.

\section{INDICADORES DEL VERDE URBANO}

El área de influencia del verde urbano disponible de Salamanca, representada como la parte de la ciudad con acceso a estos espacios, se ha determinado tal como muestra la Figura 5. Por tanto, la zona en blanco representa la parte del tejido urbano que no posee acceso a las áreas verdes, su extensión es de 253,3 ha distribuidas en dieciocho de los cuarenta y cinco barrios con los que cuenta la ciudad. Este espacio representa un $17,4 \%$ de la superficie total y se estima en 20.688 las personas que no tendrían acceso al verde urbano disponible. Esta población está mayoritariamente distribuida en tres zonas de la ciudad: al sur del río Tormes están situadas la Zona 1 y la Zona 2, en los extremos oriental y occidental del continuo urbano, mientras que la Zona 3 estaría situada en el tercio norte de la ciudad donde se concentra una gran cantidad de población. En el sur de la ciudad, la población sin acceso a la trama verde no es tanta como podría esperarse: la suma de las dos zonas tiene una extensión conjunta de 165 ha, pero únicamente vivirían 6.326 residentes censados. En esta parte de la ciudad, el caso más llamativo es el experimentado en el barrio de Buenos Aires, en la periferia suroccidental, donde sus habitantes 
deberían recorrer una distancia superior a un kilómetro para alcanzar el verde urbano más cercano. También aparecen una serie de barrios de carácter industrial, entre los que destaca el Polígono del Montalvo, situado en la Zona 2, donde se concentran a diario cientos de trabajadores que no tendrán oportunidad de acceder en las inmediaciones de su puesto de trabajo a los beneficios sociales proporcionados por las áreas verdes.

La situación del tercio norte es diferente porque los barrios situados en la parte oriental se encuentran entre los más poblados de Salamanca. Pese a la reducida extensión de la Zona 3 , cuenta únicamente con 58,4 ha, la población sin acceso al verde urbano es relativamente elevada, estimándose en cerca de 12.000 el número de residentes en esta situación. La ausencia de zonas verdes en este sector y en el resto de los barrios ubicados en el límite norte del continuo urbano, tiene su origen en una falta de planeamiento previo a la urbanización y en la consiguiente ausencia de elementos dotacionales al construir nuevos barrios en la ciudad. Esta situación se corresponde mayoritariamente con el periodo anterior a la entrada en vigor del PGOU de 1984, con el que comienza a respetarse de manera efectiva la normativa urbanística en la ciudad de Salamanca. Durante la aplicación de ese Plan General se proyectaron algunas piezas urbanas ubicadas en la periferia urbana en las que la densidad de población es relativamente baja y que cuentan con una dotación de áreas verdes muy superior a los mínimos exigidos por la normativa urbanística. Este hecho resulta destacable considerando que la búsqueda de espacios abiertos y de zonas ajardinadas, ha sido uno de los factores que aunque de manera secundaria, ha contribuido a que determinados habitantes abandonaran la vieja ciudad en busca de residencias en los municipios próximos.

Figura 5

ACCESO AL VERDE URBANO DISPONIBLE DE LA CIUDAD DE SALAMANCA

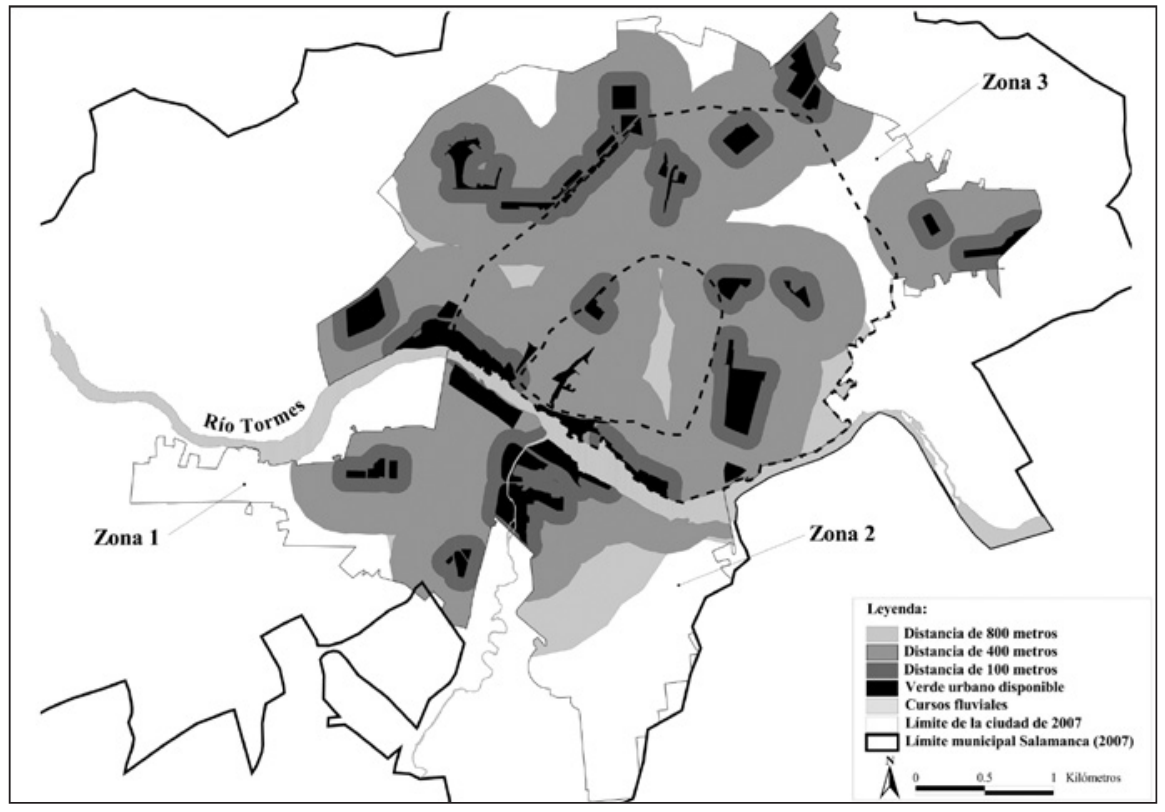

Fuente: elaboración propia a partir de la ortofotografía de 2007 del Itacyl. 
Los tres espacios verdes de más de 10 ha están situados en las proximidades del río Tormes: el Parque de los Jesuitas se encuentra ligeramente al norte de este curso fluvial, mientras que el Jardín Botánico de Huerta Otea está ubicado en la margen derecha y el Parque del Zurguén en la izquierda. Si se aplica un buffer de 800 metros desde ellos se observaría que la zona con acceso a los mismos se circunscribe a las proximidades del eje fluvial. El centro histórico apenas cuenta con un par de parques pero presenta un acceso a la trama verde relativamente bueno debido a que las grandes áreas verdes están situadas en el perímetro exterior de la cerca medieval y atenúan la escasez registrada en su interior. Sin embargo, la mitad del espacio urbano quedaría fuera de este perímetro siendo la situación especialmente grave en el tercio norte de la ciudad, en la que como ya se ha apuntado, se concentra el grueso de la población. Algunos de los barrios periféricos, tanto del tercio norte como del tercio sur, están situados a una distancia de los grandes parques que en ocasiones supera los 1.500 metros y el total de habitantes que no tienen acceso a los mismos es de 93.807 personas. La mayor concentración de zonas verdes en Salamanca se extiende a lo largo de las riberas del Tormes y de su afluente, el arroyo del Zurguén, y está compuesta por once verdes disponibles, tres en la margen izquierda y ocho en la derecha, cuyo origen mayoritario se encuentra en el mantenimiento hasta fechas relativamente recientes de actividades agrícolas en las vegas del río. Podría señalarse otra concentración verde en el cuadrante noroccidental de la ciudad que posee menores dimensiones, sumando entre ambas un $70 \%$ de la superficie del verde urbano disponible, repartida en 16 espacios. La desigual distribución provoca que la mitad de la superficie urbana se encuentre no tenga buena accesibilidad a estas dos concentraciones verdes.

$\mathrm{Al}$ analizar las zonas de juego infantil debe tenerse en cuenta que actualmente su presencia está regulada por el RUCYL, al menos en el sistema local de espacios libres públicos, y que según Magalhães (1992) este tipo de equipamientos deberían estar instalados a menos de 100 metros de su población objetivo. La superficie de la ciudad con acceso a las áreas verdes que poseen estos equipamientos equivale únicamente a una cuarta parte del continuo urbano, lo que supone una deficiencia considerable. Van Herzele y Wiedemann (2003) señalan que para determinados colectivos como son los niños o las personas con movilidad reducida, las áreas verdes de menos de una hectárea podrían desempeñar un papel destacado, por lo que sería conveniente analizar en futuros trabajos el porcentaje de visitas que los usuarios de la trama verde destina a las áreas verdes de pequeñas dimensiones.

\section{DISCUSIÓN}

Esta investigación sugiere que los índices tradicionalmente utilizados para evaluar la presencia de zonas verdes en las ciudades deben ser complementados por otro tipo de indicadores. Si se quiere evaluar la disponibilidad de uso de los espacios verdes por parte de los habitantes, habrá que incluir la posición de estas áreas en el interior del tejido urbano y utilizar la distancia máxima que un usuario estaría dispuesto a recorrer para llegar allí, determinando qué parte de la ciudad no tendría acceso a la trama verde. Sin embargo, los ratios que relacionan la superficie verde con el número de habitantes o con la extensión del tejido urbano han sido muy útiles a la hora de analizar la evolución de la ciudad y de sus espacios verdes durante los últimos cincuenta años. De esta manera se han identificado tres etapas en la evolución del verde urbano de Salamanca: la primera se extendería desde el comienzo del 
periodo de mayor crecimiento de la ciudad debido a la llegada de emigrantes de origen rural, hasta 1971, fecha en la que se alcanza el mínimo absoluto de verde urbano disponible por habitante. Los últimos gobiernos del régimen y los primeros democráticos trataron de aliviar las deficiencias heredadas en la etapa anterior, creando una serie de parques públicos hasta la aprobación del PGOU de 1984. Desde entonces se asiste a la aparición de una gran cantidad de áreas verdes fruto del cumplimiento de la normativa urbanística en las nuevas piezas urbanas y al intento de proyectar espacios verdes en el interior del tejido urbano existente.

Para contrastar los datos obtenidos se ha tomado como referencia el artículo publicado por Rodríguez y Díaz (2003), en el que se analizó la evolución histórica de una serie de parques de Madrid y se identificaron tres etapas distintas en su creación. La primera fase estaría caracterizada por la herencia de parques pertenecientes a la realeza, a la nobleza y a la Villa de Madrid. La segunda por una intensa reducción del verde urbano por habitante debido al fuerte crecimiento de la capital hasta los años setenta, fecha de inicio de la tercera etapa en la que empieza a subsanarse el déficit provocado durante las dos anteriores. Este trabajo fue publicado en 2003 y no recoge la evolución acaecida en los últimos años, pero muestra una similitud con la situación experimentada por el verde urbano de Salamanca. Sanesi y Chiarello (2006) en su estudio sobre los espacios verdes urbanos de Bari (Italia), también identificaron una evolución similar ya que en el centro histórico y en el ensanche del siglo XIX casi no había parques y jardines, mientras que en la parte edificada durante el siglo XX, que sería la más densamente poblada, sólo se empezaron a crear verdes urbanos en los últimos treinta años.

Las investigaciones llevadas a cabo en España señalan que la falta de planeamiento urbano durante las décadas de mayor crecimiento demográfico y territorial de las principales ciudades del país, provocó una serie de carencias en dotaciones urbanas en determinados barrios, que en ocasiones, no han podido ser subsanadas por las corporaciones municipales democráticas. Por ello Rodríguez y Díaz (2003) destacan el papel de los espacios verdes como indicadores de los déficits en la expansión urbana. En su trabajo, estas autoras seleccionaron únicamente las zonas verdes de la ciudad de Madrid que cumplieran con unas características concretas como tener más de 4 hectáreas, presentar límites bien definidos e individualizados y ofrecer la posibilidad de desarrollar ciertas funciones sociales. De esta manera con tan sólo 51 parques de la capital española, se analizó el $61 \%$ de la superficie verde pública de la ciudad, corroborando así la hipótesis de que un número reducido de los espacios verdes de mayores dimensiones representa un porcentaje considerable de la superficie verde mantenida por los ayuntamientos. En Salamanca los 26 verdes urbanos disponibles representan un $46 \%$ de la superficie censada por el SPYJAS, cifra que se incrementaría en un $10 \%$ si computaran todas las áreas verdes analizadas en esta investigación. Por otra parte, los índices de verde urbano per cápita son menores en la capital española que en la ciudad castellano-leonesa, ya que en Madrid sólo cinco distritos tendrían una densidad superior a $5 \mathrm{~m}^{2} /$ hab de zonas verdes, mientras que en Salamanca había once barrios con una densidad superior a $9 \mathrm{~m}^{2} / \mathrm{hab}$, siendo la media del verde urbano disponible por habitante de tan sólo $6,41 \mathrm{~m}^{2} / \mathrm{hab}$. No obstante, la comparación entre estos indicadores no es del todo adecuada porque computan áreas verdes de distintas características por no existir una definición común de verde urbano. 
Los trabajos realizados por Santana et al. (2010) en Amadora, una ciudad de la periferia de Lisboa, junto a los ya citados de Madrid y de la ciudad de Bari, han permitido distinguir una situación común del verde urbano en las ciudades del sur de Europa. En ellas se identifica un centro urbano compacto dentro del cual existen considerables deficiencias en lo que a dotación de espacios verdes se refiere, que no han comenzado a corregirse hasta fechas recientes. En oposición a este modelo se encontrarían las ciudades belgas en las que Van Herzele y Wiedemann (2003) desarrollaron su investigación y que en la mayoría de los casos contaban con una red de zonas verdes de más de 10 ha que cubrían gran parte de la trama urbana.

\section{CONCLUSIONES}

El mayor problema que presentan los trabajos que estudian los espacios verdes es la ausencia de una definición común, lo que dificulta enormemente la comparación entre publicaciones que afronten esta temática. Este artículo ha empleado una delimitación del verde urbano operativa para un ámbito territorial concreto, pero deberá ser testada en ciudades de distinta dimensión.

Al igual que mencionaban Rodríguez y Díaz (2003), es posible afirmar que las zonas verdes son verdaderos indicadores de las deficiencias urbanas, puesto que su ausencia en el interior del tejido urbano implica una falta previsión que es difícil de corregir una vez consolidadas las nuevas piezas urbanas. Se ha comprobado que con un número reducido de los parques y jardines de mayor tamaño se puede trabajar con un porcentaje significativo de la trama verde de una ciudad. Por otro lado, los índices que relacionan la superficie verde y el número de habitantes han demostrado su utilidad para realizar un análisis de la evolución del verde urbano, al permitir identificar las etapas de creación de estas dotaciones, y han constatado la estrecha relación entre el planeamiento y su creación. Concretamente, las áreas verdes han permitido identificar un modelo de ciudad mediterránea, opuesta a la del centro y a la del norte de Europa, en la que las zonas verdes han comenzado a surgir en épocas muy recientes. En el caso de Salamanca, los huertos en el interior de la ciudad han sido espacios utilizados para construir una parte del verde urbano disponible actual, lo que ha permitido aliviar algunas carencias en el interior del tejido urbano consolidado. La determinación del acceso al verde urbano ha demostrado ser un indicador muy valioso para estimar la población que no puede acceder a estas áreas, convirtiéndose en una información que podría ser de gran utilidad para las autoridades locales a la hora de proyectar la ciudad del futuro. Convendría reforzar los análisis llevados a cabo con una labor de encuestado a los usuarios del verde urbano en la que se pregunte acerca de las actividades desarrolladas en estos espacios, así como por las principales motivaciones de sus visitas.

\section{BIBLIOGRAFÍA}

AKBARI, H.; DAVIS, S.; DORSANO, S.; HUANG, J.; WINNETT, S. (1992): Cooling our communities. A guidebook on tree planting and light-colored surfacing. Washington. U.S. Environmental Protection Agency. 
BAYCAN-LEVENT, T; VREEKER, R.; NIJKAMP, P. (2004): «Multidimensional Evaluation of Urban Green Spaces: A Comparative Study on European Cities». Research memorandum, Vrije Universiteit, Faculty of Economic Business and Administration. Amsterdam: Vrije Universiteit, 1-18.

BENDIMO-RUNG, A.L.; MOWEN, A.J.; COHEN, D.A. (2005): «The Significance of Parks to Physical Activity and public health. A conceptual model». American Journal of Preventive Medicine, $\mathrm{n}^{\mathrm{o}} 28$ (252), 159-168.

BOLUND, P.; HUNHAMMAR, S. (1999): «Ecosystem services in urban areas». Ecological Economics, no 29, 293-301.

CANOSA, E.; SÁEZ, E.; SANABRIA, C.; ZAVALA, I. (2003): «Metodología para el estudio de los parques urbanos: la Comunidad de Madrid». Geofocus Revista Internacional de Ciencia y Tecnología de la Información Geográfica, nº 3, 160-185.

CHIESURA, A. (2004): «The role of urban parks for the sustainable city». Landscape and urban planning, $\mathrm{n}^{\circ} 68,129-138$.

CORONA, M.A. (2001): Las áreas verdes en el contexto urbano. Estudio de caso: ciudad de Guadalajara. México D.F..CIIEMAD.

DÍAZ, L. (1998): «Salamanca ciudad. Historia» en El libro de oro de Salamanca: un siglo en imágenes (Díaz, L., coord). Salamanca, Publines Regionales, 8-65.

DE VRIES, S.; VERHEIJ, R.; GROENEWEGEN, P. ; SPREEUWENBERG, P. (2003) : «Natural environments-healthy environments?». Environmental Planning, n 35, 1717-1731.

DWYER, J.F.; MCPHERSON, E.G.; SCHROEDER, H.W.; ROWNTREE, R.A. (1992): «Assessing the benefits and costs of the urban forest». Journal of Arboriculture, $\mathrm{n}^{\circ} 18$ (5), 227-234.

FONSECA, F.; GONÇALVES, A.; RODRIGUES, O. (2010): «Comportamentos e percepções sobre os espaços verdes na cidade de Bragança». Finisterra: Revista portuguesa de geografia, Vol.15, nº 89: 119-139.

GALINDO, M.P.; ARIAS, M.A.; DE CASTRO, R. (1997): «Usos psicosociales del espacio verde urbano». Revista de psicología social aplicada, $\mathrm{n}^{\circ} 2-3,35-52$.

GÁMEZ, V. (2005): «Sobre sistemas, tipologías y estándares de áreas verdes en el planeamiento urbano». DU \& P: revista de diseño urbano y paisaje, Vol.2, $\mathrm{n}^{\circ} 6$.

GARCÍA, A.M. (1989): «El parque urbano como espacio multifuncional: origen, evolución y principales funciones». Paralelo $37, \mathrm{n}^{\circ} 13,105-111$.

HORTELANO, L.A. (1998): «Salamanca ciudad. Desarrollo espacial» en El libro de oro de Salamanca: un siglo en imágenes (Díaz, L., coord). Salamanca, Publines Regionales, 66-121.

HORTELANO, L.A. (2000): «Cambios territoriales y retos de futuro de la ciudad de Salamanca al sur del río Tormes». Salamanca: revista de estudios, $\mathrm{n}^{\circ}$ 44, 67-81.

JUNTA DE CASTILLA Y LEÓN (2009): Reglamento de Urbanismo de Castilla y León. Valladolid. Junta de Castilla y León.

MAGALHÃES, M. (1992): Espaços verdes urbanos. Lisboa. Direcçao Geral do Ordenamento do Território.

MCPHERSON, E.G.; NOWAK, D.J.; ROWNTREE, R.A. (1994): Chicago's urban forest ecosystem: results of the Chicago urban forest climate project. Pennsylvania. U.S. Department of Agriculture. 
MIRANDA, F. (1985): Desarrollo urbanístico de postguerra en Salamanca. Salamanca. Colegio de Arquitectos de León.

NEL·LO, O.; MUÑOZ, F. (2004): «El proceso de urbanización» en Geografía humana: procesos, riesgos e incertidumbres en un mundo globalizado (Romero, J., coord). Barcelona, Ariel, 255-332.

NILSSON, K.; RANDRUP, T.; TVEDT, T. (1997): «Aspectos tecnológicos del enverdecimiento urbano» en Áreas verdes urbanas en Latinoamérica y el Caribe (Krishnamurthy, L. y Nacimiento, J., eds.) Banco Interamericano de Desarrollo, 39-81.

NOWAK, D.J.; CRANE, D.E. (2002): «Carbon storage and sequestration by urban trees in the USA». Environmental Pollution, n ${ }^{\circ} 116,381-389$.

OCHOA, J.M. (1999): La vegetación como instrumento para el control microclimático. Universitat Politècnica de Catalunya.

OCHOA, J.M. (2009): Ciudad, vegetación e impacto climático. Vilafranca del Penedés (Barcelona). Erasmus ediciones.

PRIEGO-GONZÁLEZ, C. (2008): Environmental, social and economic benefits of tree plantations for urban societies. Córdoba. IESA-CSIC.

ROCA, J. (2003): «La delimitación de la ciudad: ¿una cuestión imposible?». Ciudad y territorio: Estudios territoriales, $\mathrm{n}^{\circ} 135,17-36$.

RODRÍGUEZ, I.; DÍAZ, E.M. (2003): «Las secuelas de la ciudad negocio: los parques urbanos de Madrid» en La ciudad: nuevos procesos, nuevas respuestas. León, Universidad de León, Secretariado de Publicaciones y Medios Audiovisuales, 193-204.

SÁNCHEZ-BARBUDO, M.C. (1989): «El método de trabajo de campo y laboratorio en las ciencias naturales de EGB». Aula: Revista de Pedagogía de la Universidad de Salamanca, $\mathrm{n}^{\circ}$ 2: 89-91.

SANESI, G.; CHIARELLO, F. (2006): «Residents and urban green spaces: The case of Bari.» Urban Forestry Urban Greenning, 4(3-4): 125-134.

SANTANA, P. ; COSTA, C.; SANTOS, R. ; LOUREIRO, A. (2010): «O papel dos Espaços Verdes Urbanos no bem-estar e saúde das populações». Revista de Estudos Demográfi$\cos , \mathrm{n}^{\circ} 48,6-33$.

SANTANA, P.; NOGUEIRA, H.; SANTOS, R.; COSTA, C. (2007): «Avaliação da Qualidade Ambiental dos Espaços Verdes Urbanos no Bem-estar e na Saúde» en A Cidade e a Saúde (Santana, P., coord). Coimbra, Almedina, 219-237.

SEGOVIA, O.; NEIRA, H. (2005): «Espacios públicos urbanos: una contribución a la identidad y confianza social y privada». Revista INVI, Vol. 20, 55, 126-182.

SENABRE, D. (2003a): «Desarrollo urbano y urbanística del municipio de Salamanca en el siglo XX» en Scripta Nova, vol. VII, num. 146, 1 de agosto de 2003. Disponible en: http://www.ub.edu/geocrit/sn/sn-146(139).htm

SENABRE, D. (2003b): «La construcción de viviendas en Salamanca (1984-2002)» en Scripta Nova, vol. VII, num. 146, 1 de agosto de 2003. Disponible en: http://www.ub.edu/geocrit/sn/sn-146(092).htm

TERÁN, F. (1978): Planeamiento urbano en la España contemporánea: historia de un proceso imposible. Barcelona. Gustavi Gili.

ULRICH, R.S. (1984): «View thought a window may influence recovery from surgery». Science, New Series, ${ }^{\circ} 224,420-421$. 
ULRICH, R.S.; SIMONS, R.F.; LOSITO, B.D.; FIORITO, E.; MILES, M.A.; ZELSON, M. (1991): «Stress recovery during exposure to natural and urban environments». Journal of Environmental Psychology, $\mathrm{n}^{\circ}$ 11, 201-230.

UNIÓN EUROPEA (2003): European Common Indicators. Towards a Local Sustainability Profile. Ambiente Italia. Istituto Di Ricerche.

VAN HERZELE, A.; WIEDEMANN, T. (2003): «A monitoring tool for the provision of accessible and attractive urban green spaces». Landscape Urban Plan, n 63, 109-126.

VILLAR, J. (2000): «De ciudad encorsetada a desparramada metrópolis: Salamanca en el siglo XX». Salamanca: revista de estudios, $\mathrm{n}^{\circ} 45,189-220$.

VILLAR, J.; ALONSO, J.L.; SÁNCHEZ, J.L. (1995): «Salamanca: etapa de gran crecimiento» en Salamanca y sus comarcas (Cabero, V., coord.). Madrid, Editorial Mediterráneo, 337-344.

VILLAR, J.; BURRIEZA, B. (1995): «Salamanca: la consolidación como capital de provincia» en Salamanca y sus comarcas (Cabero, V., coord.). Madrid, Editorial Mediterráneo, 329-336.

VILLAR Y MACÍAS, M. (1887): Historia de Salamanca. Libro IX, Desde la Guerra de la Independencia hasta nuestros días. Salamanca. Librería Cervantes. 
